\title{
Received Power Attenuation Analysis Based on Wavelet for Reflection-Style Optical Antenna Deformations in Free-Space Laser Communications
}

\author{
Wanqing Xie, Liying Tan, Jing Ma, and Yang Liu \\ National Key Laboratory of Tunable Laser Technology, Harbin Institute of Technology, Harbin 150001, China \\ Correspondence should be addressed to Wanqing Xie, zwq.xie@gmail.com
}

Received 5 January 2011; Revised 1 March 2011; Accepted 24 March 2011

Academic Editor: Mandeep Jit Singh

Copyright (C) 2011 Wanqing Xie et al. This is an open access article distributed under the Creative Commons Attribution License, which permits unrestricted use, distribution, and reproduction in any medium, provided the original work is properly cited.

\begin{abstract}
Diversified deformations on reflection-style optical antennas caused by processing technique and complex outer-space environment in free-space laser communications are modeled by wavelet analysis. Influence due to the deformations on received power is studied by distortion attenuation factor based on the model. Theoretical and numerical analysis shows that distortion attenuation factor increases with shift factor of the model, decreases with dilation factor of the model, and decreases with irregular fluctuations with wavelet coefficient of the model. To the special case that the deformation can be well approximated to a constant, distortion attenuation factor varies periodically with the linear increase of the deformation coefficient. A reference for the requirement on the precision of optical antenna is proposed, and a method to reduce the effect of deformation is recommended. It is hoped that the study can be used in the design of free space laser communication systems.
\end{abstract}

\section{Introduction}

Free space laser communication is a kind of technique which employs laser as the carrier to transmit signals between two terminals. With its potential commercial and military applications due to smaller size and weight of the terminal, less power consumption, larger data rate, denser satellite orbit population, and greater immunity to interference which compare to microwave communications, the research of this technique has become a hot spot [1-6]. Since intersatellite laser communication system works with the limitation of optical diffraction, communication distance and photoelectric detection, high quality laser beam, and high precision optical terminals are demanded. However, wavefront distortions can be induced by the precision of optical devices, outer space temperature variation, contamination, and space radiation. Slight wave-front distortions can have significant effect on receiving terminal due to small beam divergence and ultralong distance of the communication link [7-9]. To deal with the problem, many studies have been focusing on possible solutions to decrease the effect of distortions, such as the optical phased array $[10,11]$, while others have been researching on the influence of deformations on the system.

In 2001, Toyoshima et al. described wave-front aberrations by Zernike polynomials in circle region to study the influence of deformations in free space laser communications [12]. In 2005, Sun et al. developed the description to annular region by using the Gram-Schmidt orthogonalization procedure to modify the original Zernike polynomials because the optical antenna for satellite laser communication systems regularly encounters centrally obscured pupils, such as the Cassegrain telescope system [13]. Due to various kinds of complicated affecting factors, the styles of the deformations are uncertain and with strong randomness. The deformations may be localized or asymmetric. Instead of Zernike polynomials, wavelet analysis is employed in the paper to model diversified deformations which may appear on the optical antenna. It is a method which has been widely applied for the description of nonstable signals, especially for surface roughness $[14,15]$. With the multiresolution characteristic of wavelet analysis, deformations can be decomposed into several subdeformations by which the original ones can be localized in both space and frequency 


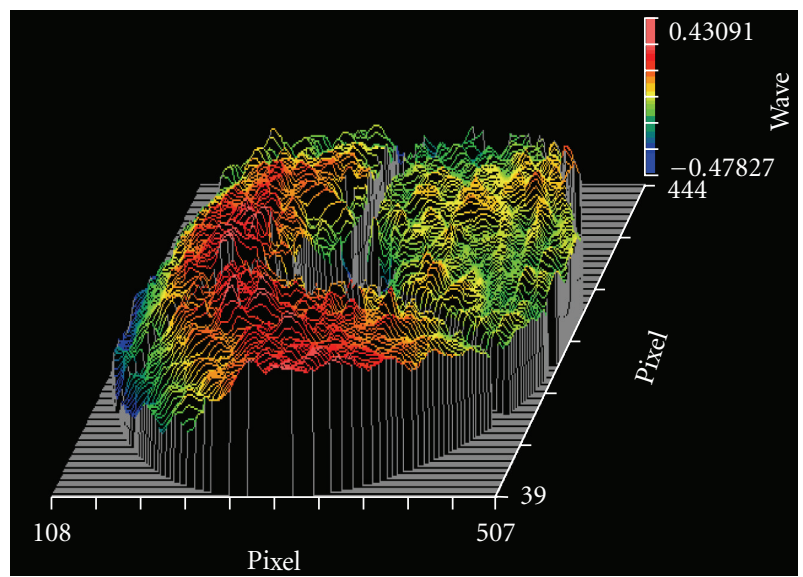

FIGURE 1: Example antenna deformation caused by temperature variation detected by Zygo interferometer.

domains. Consequently, diversified deformations can be modeled by the space-frequency atom of wavelet analysis. For deformations in the transmitter can degrade the received signal on the receiver plane, or even increase the bit error rate of the system [12], the purpose of the study is to estimate the influence of generalized deformations of optical antennas on system received power with the model.

This paper proposes wavelet model for antenna deformations and shows the phase delay for deformed reflectionstyle antenna with the model. The influence of generalized deformations on received power in free space laser communication systems is discussed with the parameters of the model. In addition, a principle for deformed optical devices selection is given, a reference for machining precision of optical antennas is provided and a method to reduce the influence of deformations is recommended.

The rest of the paper is organized as follows: deformation model based on wavelet is in Section 2, received power attenuation analysis with the model is in Section 3, Section 4 is for numerical results, and Section 5 is conclusion.

\section{Antenna Deformation Analysis Based on Wavelet}

Owing to the limitation of processing technique and the effect of space environment, deformations are extremely likely to be induced on the optical antenna. Figure 1 is an example of deformation on a reflection-style optical antenna caused by the variation of temperature. As shown in Figure 1, the deformation is asymmetric and irregular. A proper method which can express the deformation with efficiency is required.

As any function in the energy limited space can be expended by wavelets [16], deformed optical antenna $D(x, y)$ can be depicted by the linear combination of twodimensional discrete wavelets as

$$
D(x, y)=\sum_{a} \sum_{(b, d)} \alpha_{a ; b, d} \psi_{a ; b, d}(x, y)
$$

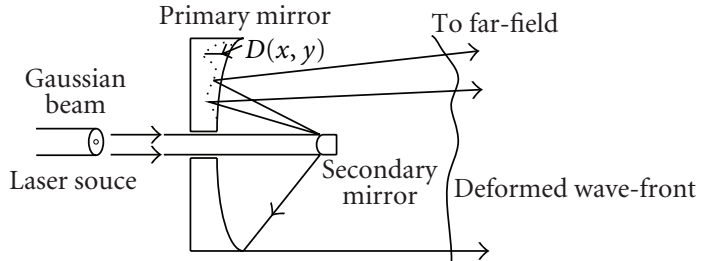

FIGURE 2: Reflection-style antenna with and without deformation.

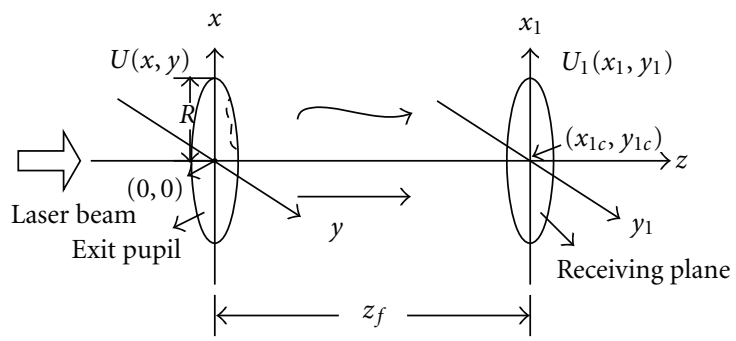

Figure 3: Definition of the coordinate system.

where $\psi_{a ; b, d}(x, y)=(1 / a) \psi((x-b) / a,(y-d) / a)$ is the wavelet function. $a$ is the dilation factor, and it must be a nonzero real number. With the increase of $a$, the shape of the subdeformation depicted by wavelet becomes lower and fatter. If considered in the frequency domain, larger $a$ depicts the subdeformation with lower frequency band. $(b, d)$ is the shift factor which determines the location of the subdeformation. With different $(a ; b, d)$, the whole deformation can be analyzed in a local area near $(b, d)$ with dilation factor $a . \alpha_{a ; b, d}$ is the wavelet coefficient which can show the similarity between each sub-deformation $\psi_{a ; b, d}(x, y)$ and the whole deformation $D(x, y)$. Moreover, $\left|\alpha_{a ; b, d}\right|^{2}$ stands for the energy of $D(x, y)$ distributed at $(a ; b, d)$.

The diagram of the reflection-style antenna with and without irregular deformation is shown in Figure 2.

By the wavelet expression as shown in (1), localized and global deformations with irregular shapes can be expressed. When the transmitted Gaussian laser beam encounters the deformation on the antenna, the wave-front of the laser beam will be disturbed and the phase of the original wave-front will be changed. The phase delay caused by the deformation is proportional to the optical path difference $\Delta L$. For the antenna shown in Figure 2, $\Delta L$ due to the deformation is $2 D(x, y)$. Then, the phase delay can be express as

$$
\Phi(x, y)=k \Delta L(x, y)=k 2 D(x, y),
$$

where $k=2 \pi / \lambda$ is wave number $\lambda$ is wave length.

Input (1) to (2), and make the wavelet transform to the delayed phase

$$
W_{a ; b, d}[\Phi(x, y)]=\frac{4 \pi}{\lambda} \alpha_{a ; b, d} \psi_{a ; b, d}(x, y),
$$

where $W_{a ; b, d}[\Phi(x, y)]$ is the wavelet model for the phase delay caused by generalized distortions. 


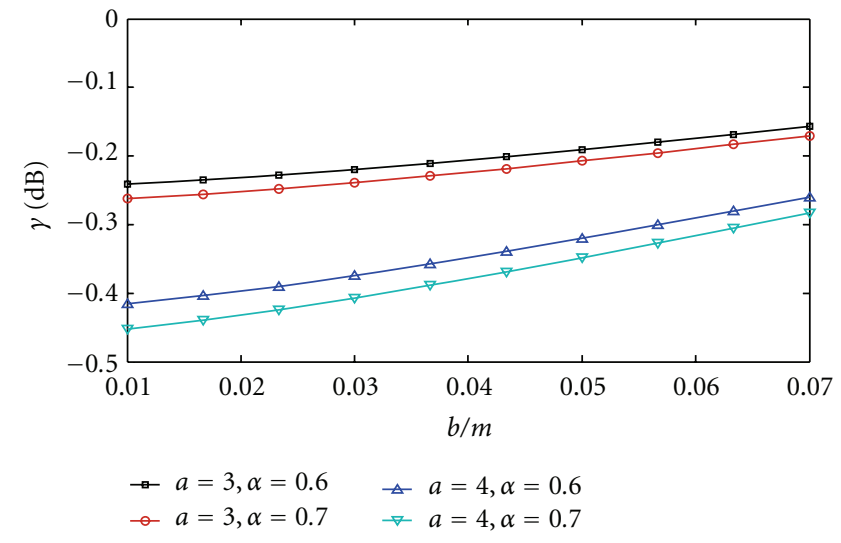

(a) $\psi_{\text {Shannon }} \gamma-b$

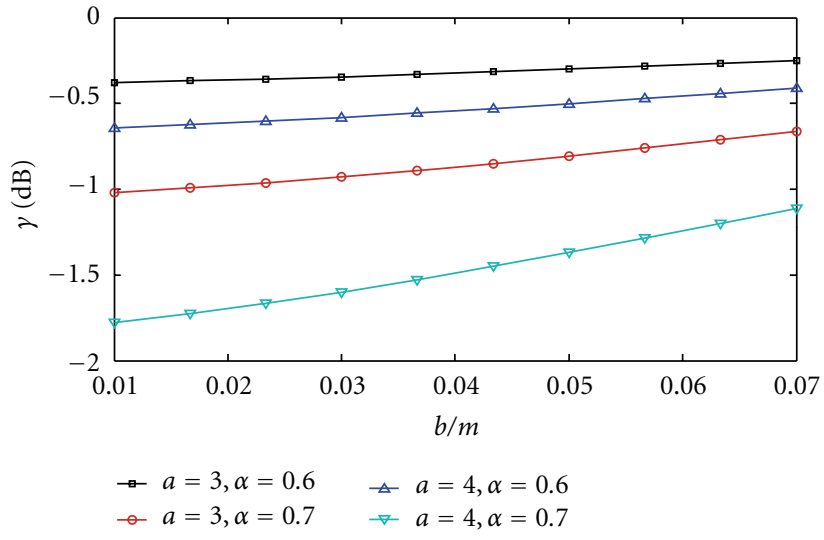

(b) $\phi_{\text {Haar }} \gamma-b$

FIGURE 4: Distortion attenuation factor $\gamma$ with the consideration of shift factor $b$.

\section{Received Power Attenuation Analysis}

In free space laser communication systems, the signal is transmitted by laser beam from one communication terminal to the other. Significant influence on the optical field distribution after long distance transmission can be induced by fine deformations on the transmitter optical antenna. This paper focuses on the influence of antenna deformations, which are with the order of wavelength, on received power.

Diagram of the definition of the coordinate system is shown in Figure 3. The transmitted laser beam passes through the exit pupil to far-field receiving plane. The wavefront of transmitted laser beam will be disturbed when it encounters the deformation on the transmitter, and the optical field distribution on the receiving plane will be affected.

$U(x, y)$ is the transmitted Gaussian laser beam. The optical field $U^{\prime}(x, y)$ right after the deformed antenna can be expressed with the wavelet model as

$$
U_{a ; b, d}^{\prime}(x, y)=M(x, y) U(x, y) \exp \left[j W_{a ; b, d}[\Phi(x, y)]\right]
$$

where $M(x, y)$ is the aperture function of the antenna

$$
M(x, y)= \begin{cases}1 & \left(D_{1} / 2\right)^{2} \leq x^{2}+y^{2} \leq\left(D_{2} / 2\right)^{2} \\ 0 & \text { otherwise }\end{cases}
$$

where $D_{1}$ and $D_{2}$ are the diameters of the secondary and primary mirrors, respectively. The optical intensity on the receiver can be written as

$$
\begin{aligned}
& I_{a ; b, d}\left(x_{1}, y_{1}\right) \\
& \quad=\frac{A^{2}}{\lambda^{2} z_{f}^{2}}\left|\iint U_{a ; b, d}^{\prime}(x, y) \exp \left[-\frac{j k}{z_{f}}\left(x x_{1}+y y_{1}\right)\right] d x d y\right|^{2},
\end{aligned}
$$

where $z_{f}$ is the distance between the two communication terminals. Received power $P$ discussed in the paper is given by

$$
P_{a ; b, d}=\iint_{S} I_{a ; b, d}\left(x_{1}, y_{1}\right) d s,
$$

where $S$ is the area of the receiver. Though wave-front deformation exists, the distorted wave-front can be seemed as a plane wave on the receiver for the aperture of the receiver is small enough. Hence, the intensity of the receiver beam can be approximated to a constant over the full area of the aperture. Received power $P$ with and without deformation in the transmitter proportionally depends on the light intensity at the central point $\left(x_{1 c}, y_{1 c}\right)$ of the receiving plane. To quantify the influence of deformation on received power, distortion attenuation factor $\gamma$ is proposed as

$$
\gamma=10 \log \frac{P_{a ; b, d}}{P_{0}}=10 \lg \frac{I_{a ; b, d}\left(x_{1 c}, y_{1 c}\right)}{I_{0}\left(x_{1 c}, y_{1 c}\right)},
$$

in which $P_{a ; b, d}$ and $P_{0}$ are the received power with and without deformation, respectively. $I_{a ; b, d}\left(x_{1 c}, y_{1 c}\right)$ and $I_{0}\left(x_{1 c}, y_{1 c}\right)$ are the light intensity at the central point of the receiving plane with and without deformation, respectively.

It can be seen from (8) that the distortion attenuation factor $\gamma$ is determined by dilation factor $a$, shift factor $(b, d)$ and wavelet coefficient $\alpha$ of the model with a selected wavelet $\psi$.

With the increase of shift factor $(b, d)$, the location of the subdeformation moves away from center to the edge of the antenna. For the transmitted Gaussian laser beam $U(x, y)$ in (4), the light intensity distribution is in accord with Gaussian function. Weaker light intensity is distributed with farther distance away from the center of the beam. When the deformation is totally in the aperture of the antenna without any blocking of the secondary mirror of the reflection-style optical antenna, the effect on received power $P$ is suppressed by the light intensity of Gaussian laser beam with the increase of shift factor $(b, d)$. Consequently, according to (8), distortion attenuation factor $\gamma$ increases 


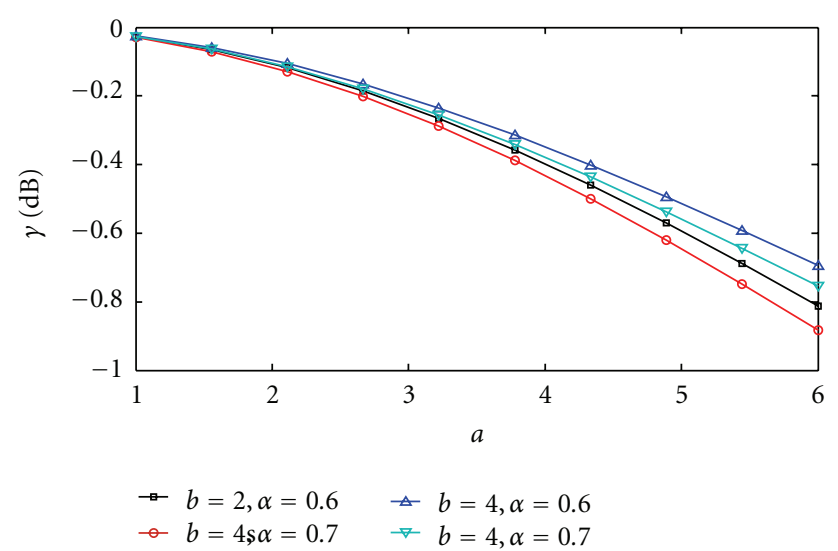

(a) $\psi$ Shannon $\gamma-a$

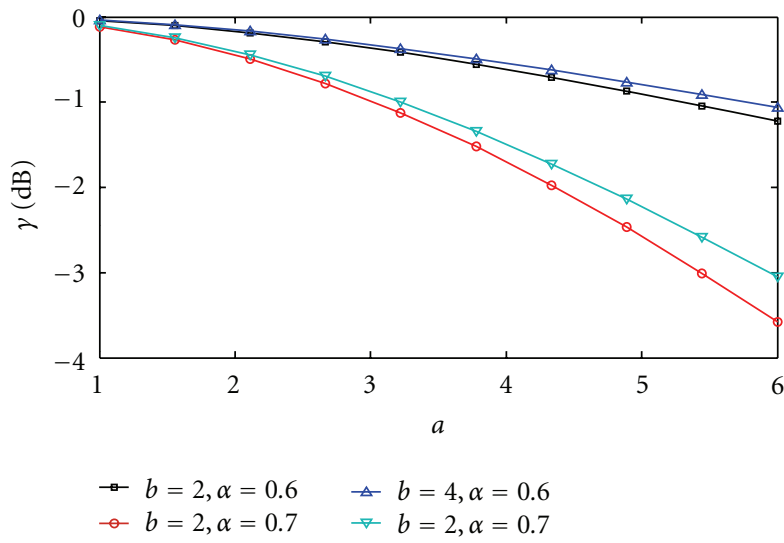

(b) $\phi_{\text {Haar }} \gamma-a$

FIGURE 5: Distortion attenuation factor $\gamma$ with the consideration of dilation factor $a$.

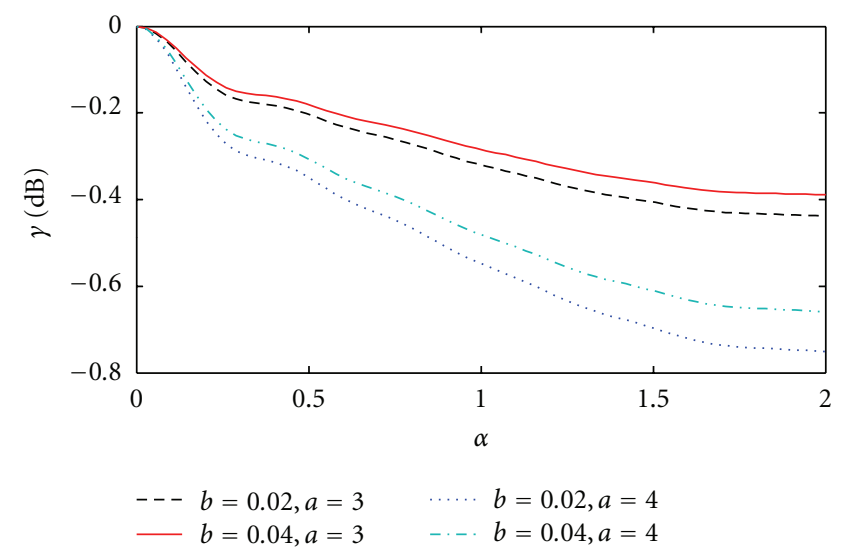

(a) $\psi_{\text {Shannon }} \gamma-\alpha$

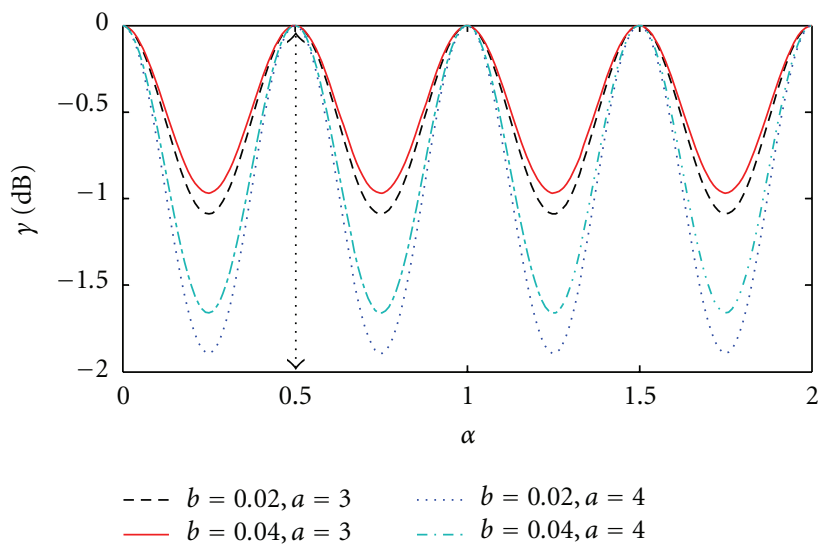

(b) $\phi_{\text {Haar }} \gamma-\alpha$

FIGURE 6: Distortion attenuation factor $\gamma$ with the consideration of $\alpha$.

with shift factor $(b, d)$. When the deformation is part in the obscuration area, the deficiency induced by the distortion can be reduced until the whole expose to the exit pupil.

With the increase of dilation factor $a$, the shape of the subdeformation expressed by the wavelet model becomes fatter and lower. If considered in the frequency domain, the frequency band affected by the wavelet filter becomes lower with the increase of $a$. As in (6), larger influence is induced on the light intensity in the receiver. Consequently, distortion attenuation factor $\gamma$ decreases with dilation factor $a$.

Generally, $\exp (j k \alpha)$ is a periodic function with the linear increase of $\alpha$, where $k$ is a nonzero constant. To the wavelet model in (4), $\exp \left[j 4 \pi \alpha_{a ; b, d} \psi_{a ; b, d}(x, y) / \lambda\right]$ is no longer a periodic function with the linear increase of $\alpha_{a ; b, d}$, because $\psi_{a ; b, d}(x, y)$ cannot be properly approximated to a constant according to the characteristic of wavelet [16]. The energy of the subdeformation increases with the increase of $\alpha_{a ; b, d}$, which will cause the decrease of received power. Hence, distortion attenuation factor $\gamma$ decreases with irregular fluctuations with the increase of wavelet coefficient $\alpha_{a ; b, d}$.
However, regardless of the characteristic of wavelet, if the deformation can be well approximated to a constant $C$, the exponential function can be rewritten as $\exp [j 4 \pi \alpha C / \lambda]$. Hence, in this particular situation, with the linear increase of $\alpha$, which is named as the deformation coefficient for this particular situation, distortion attenuation factor $\gamma$ fluctuates periodically.

\section{Numerical Results and Analysis}

To investigate the influence of generalized deformations on received power numerically with the model presented in the paper, the parameters for calculation process are set as follow: in (3) and (6), the wave length $\lambda$ is $800 \mathrm{~nm}$, and the distance $z_{f}$ between two communication terminals is $50,000 \mathrm{~km}$. In (5), the diameters $D_{1}$ and $D_{2}$ of the secondary and primary mirrors of the reflection-style optical antenna are $0.25 \mathrm{~m}$ and $0.02 \mathrm{~m}$, respectively. In calculation, all the deformations are considered totally in the aperture of the antenna, and one of the shift factors $d$ is set to zero for the symmetry of lens. 
TABLE 1: Comparison and results from $\psi_{\text {Shannon }}$ and $\phi_{\text {Haar }}$.

\begin{tabular}{lcc}
\hline & $\psi_{\text {Shannon }}$ & $\phi_{\text {Haar }}$ \\
\hline Name & Wavelet function of Shannon & Scale function of Haar \\
Mean value & 0 & 1 \\
$\begin{array}{l}\text { Applicable } \\
\text { deformation }\end{array}$ & Deformations cannot properly approximated to a constant & Deformations can be well approximated to a constant \\
$\gamma-b$ & Increase & Increase \\
$\gamma-a$ & Decrease & Decrease \\
$\gamma-\alpha$ & Decrease with irregular fluctuation & Fluctuates periodically \\
\hline
\end{tabular}

Numerical results with various kinds of wavelet functions $\psi$ have been investigated for the diversity of deformations. Typical results from Shannon wavelet $\psi$ Shannon which has the ability of effective segmentation in the frequency domain $[17,18]$ are given in the paper. Similar results can be obtained with other wavelets. Besides, for the special case that the deformation can be well approximated to a constant, the scaling function of Haar $\phi_{\text {Haar }}$, which is a constant and has the ability of effective segmentation in space domain $[19,20]$, is selected for the calculation process.

The result for distortion attenuation factor $\gamma$ with the consideration of shift factor $b$ is shown in Figure 4. Figure $4(\mathrm{a})$ is the result from Shannon wavelet $\psi$ shannon, and Figure 4(b) is the result from the scaling function of Haar $\phi_{\text {Haar }}$. For both situations, distortion attenuation factor $\gamma$ increases with shift factor $b$, which means the influence of deformation decreases with the shift factor of the model. The numerical results are coincidence with the theoretical analysis in the previous section.

Figure 5 is the result for the variation of distortion attenuation factor $\gamma$ with the consideration of dilation factor

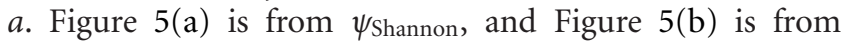
$\phi_{\text {Haar }}$. It can be observed that for both of the situations, distortion attenuation factor $\gamma$ decreases with dilation factor $a$, which means the influence of deformation on received power increases with the dilation factor of the model. The numerical results are consistent with the theoretical analysis above.

Figure 6 shows the variation of distortion attenuation factor $\gamma$ with the consideration of coefficient. Figure 6(a) is the results from $\psi_{\text {Shannon, and Figure } 6(\mathrm{~b}) \text { is the results }}$ from $\phi_{\text {Haar }}$ It can be seen from Figure $6(\mathrm{a})$ that distortion attenuation factor decreases with irregular fluctuations with the increase of wavelet coefficient. While in Figure 6(b), with the linear increase of the deformation coefficient, distortion attenuation factor fluctuates periodically. Considering the deformation with the unit of wavelength, period $T$ can be calculated from (4) as

$$
T=\frac{2 \pi}{W_{a ; b, d}[\Phi(x, y)]}=\frac{2 \pi}{4 \pi \phi_{\text {Haar }}}=\frac{1}{2},
$$

where $\phi_{\text {Haar }}(x, y)=1$, and the period $T$ does not depend on other parameters of the model.

To summarize the results of the study, Table 1 shows the related information and results about the wavelet function of Shannon $\psi_{\text {Shannon }}$ and the scale function of Haar $\phi_{\text {Haar }}$.
From the theoretical and numerical analysis above, if optical antenna with deformation has to be selected, to maximally maintain the received power in free space laser systems, principle for deformed antenna selection is as follows: we should select the deformed antenna with smaller dilation factor, smaller wavelet coefficient, and larger shift factor. To the machining precision of optical antenna, deformations with larger dilation factor, larger wavelet coefficient, and smaller shift factor should be avoided. As the distortion attenuation factor decreases with the increase of dilation factor, the influence may be reduced by enlarging the aperture of the optical antenna.

\section{Conclusion}

Wavelet model has been proposed in the paper for diversified deformations on optical antenna in free space laser communications. This model can express generalized deformations locally and globally with efficiency. The effect of deformations on received power has been studied by distortion attenuation factor with the parameters of the model. Theoretical analysis and numerical results show that distortion attenuation factor increases with shift factor of the model, decreases with dilation factor of the model, and decreases with irregular fluctuations with wavelet coefficient of the model. Specially, when the deformation can be well approximated to a constant, distortion attenuation factor will fluctuate periodically with the linear increase of deformation coefficient. A principle for deformed optical antenna selection and a reference for machining precision of optical antenna were proposed. The study will contribute to the design of free space communication systems and help to improve the performance of the systems.

\section{Acknowledgments}

This work is supported by the National Natural Science Foundation of China (Grant no. 10904026). All the authors are grateful to the editor and to the anonymous referees for their helpful comments and suggestions.

\section{References}

[1] J. Ma, M. Li, L. Y. Tan, Y. P. Zhou, S. Y. Yu, and C. Che, "Space radiation effect on EDFA for inter-satellite optical communication," Optik, vol. 121, no. 6, pp. 535-538, 2010. 
[2] L. Wan, L. Liu, and J. Sun, "On-ground simulation of optical links for free-space laser communications," Optik, vol. 121, no. 3, pp. 263-267, 2010.

[3] M. H. Mahdieh and M. Pournoury, "Atmospheric turbulence and numerical evaluation of bit error rate (BER) in free-space communication," Optics and Laser Technology, vol. 42, no. 1, pp. 55-60, 2010.

[4] Q. Yang, L. Tan, and J. Ma, "Doppler characterization of laser inter-satellite links for optical LEO satellite constellations," Optics Communications, vol. 282, no. 17, pp. 3547-3552, 2009.

[5] S. Arnon, S. Rotman, and N. S. Kopeika, "Beam width and transmitter power adaptive to tracking system performance for free-space optical communication," Applied Optics, vol. 36, no. 24, pp. 6095-6101, 1997.

[6] D. Kedar and S. Arnon, "Urban optical wireless communication networks: the main challenges and possible solutions," IEEE Communications Magazine, vol. 42, no. 5, pp. S2-S7, 2004.

[7] B. M. Levine, E. A. Martinsen, A. Wirth et al., "Horizontal line-of-sight turbulence over near-ground paths and implications for adaptive optics corrections in laser communications," Applied Optics, vol. 37, no. 21, pp. 4553-4560, 1998.

[8] B. R. Strickland, M. J. Lavan, E. Woodbridge, and V. Chan, "Effects of fog on the bit-error rate of a free-space laser communication system," Applied Optics, vol. 38, no. 3, pp. 424-431, 1999.

[9] V. N. Mahajan, "Line of sight of an aberrated optical system," The Journal of the Optical Society of America A, vol. 2, pp. 833836, 1985.

[10] A. Polishuk and S. Arnon, "Communication performance analysis of microsatellites using an optical phased array antenna," Optical Engineering, vol. 42, no. 7, pp. 2015-2024, 2003.

[11] W. M. Neubert, K. H. Kudielka, W. R. Leeb, and A. L. Scholtz, "Experimental demonstration of an optical phased array antenna for laser space communications," Applied Optics, vol. 33, no. 18, pp. 3820-3830, 1994.

[12] M. Toyoshima, N. Takahashi, T. Jono, T. Yamawaki, K. Nakagawa, and A. Yamamoto, "Mutual alignment errors due to the variation of wave-front aberrations in a free-space laser communication link," Optics Express, vol. 9, no. 11, pp. 592602, 2001.

[13] J. Sun, L. Liu, M. Yun, and L. Wan, "Mutual alignment errors due to wave-front aberrations in intersatellite laser communications," Applied Optics, vol. 44, no. 23, pp. 49534958, 2005.

[14] V. Niola, G. Nasti, and G. Quaremba, "A problem of emphasizing features of a surface roughness by means the discrete wavelet transform," Journal of Materials Processing Technology, vol. 164-165, pp. 1410-1415, 2005.

[15] S. Pal, S. K. Ghatak, S. De, and S. DasGupta, "Evaluation of surface roughness of a plasma treated polymeric membrane by wavelet analysis and quantification of its enhanced performance," Applied Surface Science, vol. 255, no. 5, pp. 2504-2511, 2008.

[16] D. B. Percival and A. T. Walden, "Discrete wavelet transform," in Wavelet Methods for Time Series Analysis, pp. 135-137, Cambridge University Press, Cambridge, UK, 2000.

[17] C. Cattani, "Shannon wavelets theory," Mathematical Problems in Engineering, vol. 2008, Article ID 164808, 24 pages, 2008.

[18] Y. Q. Xu and J. A. Liu, "Shannon wavelet chaotic neural network with chaotic noise," in Proceedings of the 9th Wuhan International Conference on E-Business, pp. 2133-2140, 2010.
[19] J. Majak, M. Pohlak, and M. Eerme, "Application of the haar wavelet-based discretization technique to problems of orthotropic plates and shells," Mechanics of Composite Materials, vol. 45, no. 6, pp. 631-642, 2009.

[20] A. Petsa and T. Sapatinas, "Adaptive quadratic functional estimation of a weighted density by model selection," Statistics, vol. 44, no. 6, pp. 571-585, 2010. 

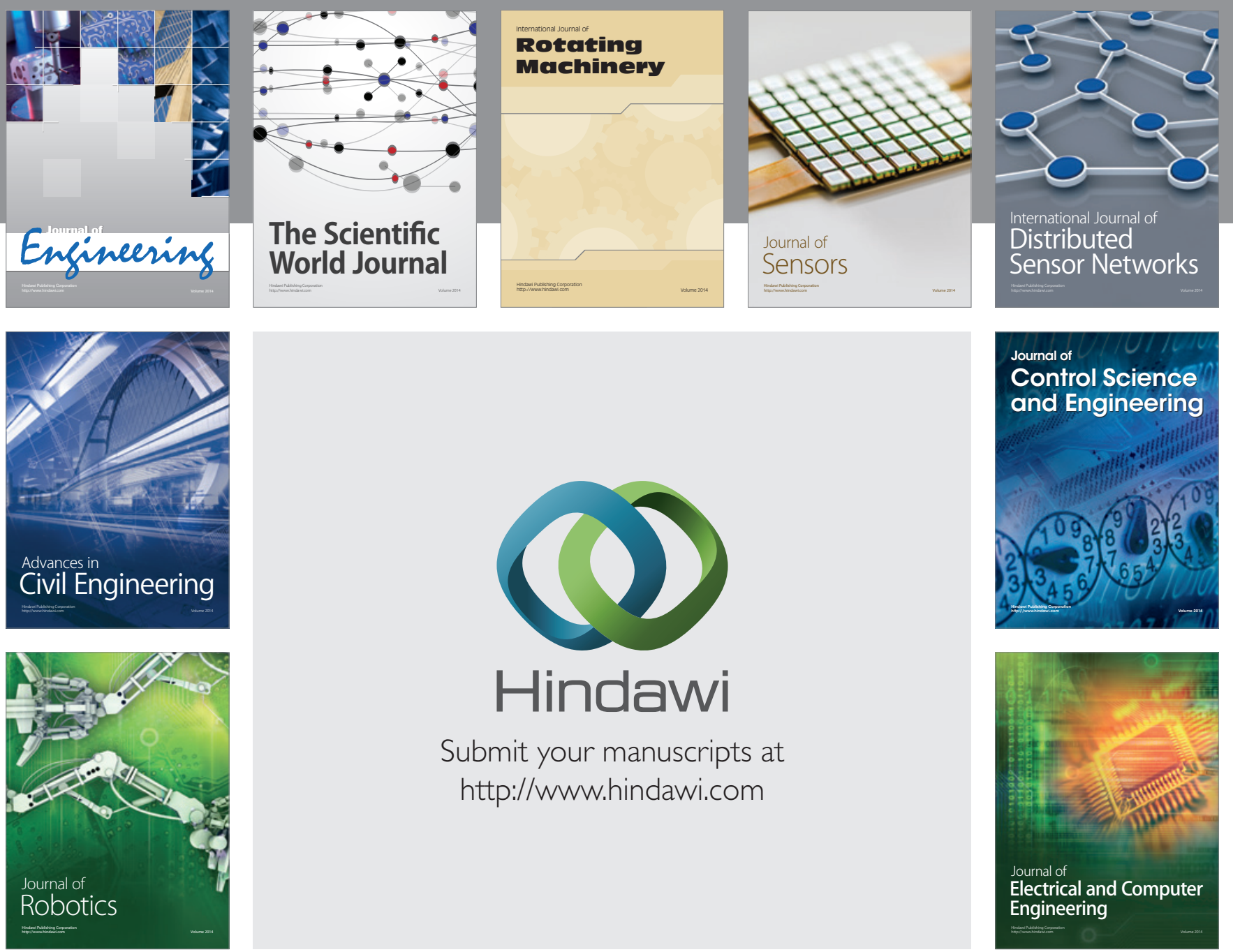

Submit your manuscripts at

http://www.hindawi.com
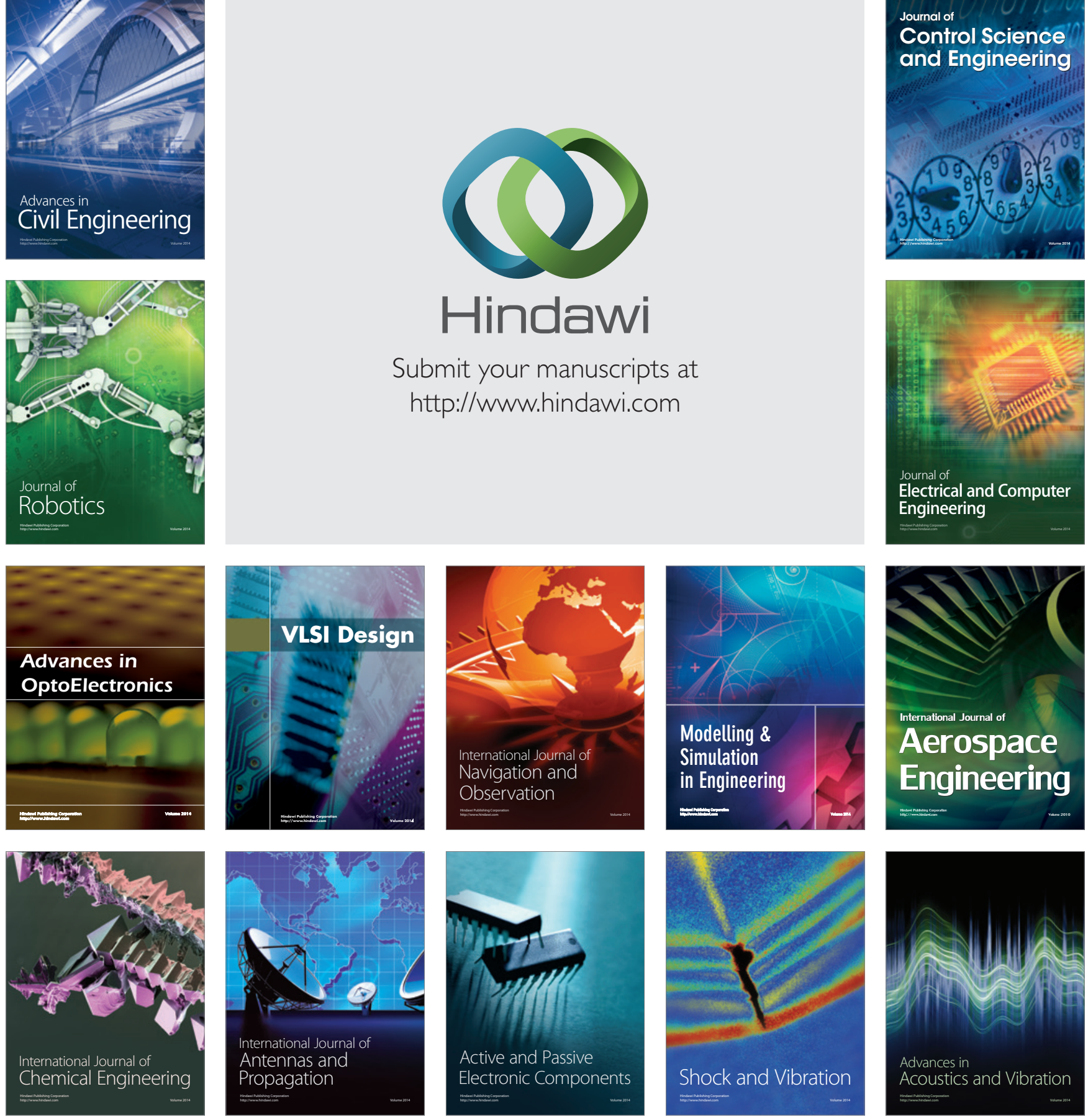